\title{
Computational Nanophotonics: Model Optical Interactions and Transport in Tailored Nanosystem Architectures

\author{
DOE Grant DE-FG02-03ER15486
}

\author{
PI: Mark Stockman \\ Department of Physics and Astronomy, Georgia State University, Atlanta, GA \\ 30303
}

E-mail: mstockman@gsu.edu, URL: http://www.phy-astr.gsu.edu/stockman

Final Report 2003-2007

\section{Program Scope}

The program is directed toward development of new computational approaches to photoprocesses in nanostructures whose geometry and composition are tailored to obtain desirable optical responses. The emphasis of this specific program is on the development of computational methods and prediction and computational theory of new phenomena of optical energy transfer and transformation on the extreme nanoscale (down to a few nanometers).

\section{Progress Achieved}

\subsection{Coherent Control of Ultrafast Energy Localization at Nanoscale [1, 2]}

Our research has significantly focused on problem of controlling localization of the energy of ultrafast (femtosecond) optical excitation on the nanoscale. We have proposed and theoretically developed a general approach to solving this fundamental problem [3-9]. It is difficult to overestimate possible applications of this effect, including nano-chip computing, nanomodification (nanolithography), and ultrafast nano-sensing. A major obstacle in using these ideas of the coherent control at the nanoscale has been the absence of efficient numerical methods to compute the waveform of the excitation pulses required to localize the optical energy at a given nanosite at a required time. Recently we have developed such a method based on the idea of the time reversal $[1,2]$. Numerically, this method requires the computation of the retarded dyadic Green's functions for the system. It is sufficiently efficient with respect to the CPU time, very stable but requires very large RAM memory (in the tens to hundred Gigabyte range).

Following our pioneering work, there has recently been an explosion of activity on both theoretical [10-13] and experimental [14-18] investigations of the ultrafast coherent control on the nanoscale. This field will rapidly grow into one of the most important in the nanoscience with application to the nanoscale computations, sensing, spectroscopy, etc. It will require our increased attention to stay at the forefront. We are currently investigating this new time-reversal approach to include the polarization of the excitation pulses.

\subsection{Spatio-Temporal Coherent Control at Nanoscale [19]}

We have developed a new approach to dynamically control the nanoscale distribution of optical energy [19]. This approach amalgamates three principles: adiabatic compression [20], spatial coherent control (optical aperture synthesis) [21-23], and the temporal coherent control at the nanoscale [1-4, 6, 8]. An example of the system that can be used for the spatio-temporal control, we consider a plasmon-polaritonic tapered waveguide, in particular, a silver wedge whose maximum thickness is in a 30-nm range, and the minimum thickness is on order of a few (2-3) nanometers. There are nanoparticles (or nano-indentations) situated at the thick edge, on which we will focus the output a spatio-temporal modulator. These nanoparticles will couple the incident radiation to the surface plasmon polaritons (SPPs) propagating in the plane of the wedge toward its thick edge. This is illustrated in Fig. 1(a) where the coupling nanoparticles are shown by circles and the rays of the SPPs are indicated by lines. The phases of the excitation waves focused at the individual particles define the 
curvature of the SPP wave front in the plane shown by concave curves. These wavefronts are adjusted by the phase modulation in such a way that these SPP rays converge at a nanofocus at the thin edge of the wedge [Fig. 1 (a) and (b)]. To have the pulses of the SPP coming along each ray to coincide at the nanofocus and produce there an ultrashort (a few fs) pulse of the local fields, each of the excitation pulses should be modulated in time as shown in Fig. 1(c).

The problem of the adiabatic concentration is extremely complicated numerically: it includes the three spatial coordinates and time. There are large ratios of the maximum to minimum scales present in each dimension. Its full numerical solution at this time using, e.g., finite difference time-domain method would be too complex. Therefore as the first step in solving this problem and to obtain a reasonable initial field, we have employed a WKB approximation, which is expected to work well precisely for these conditions of the large maximum/minimum scale ratios. This is how the solution shown in Fig.1 has been obtained [19]. In the future, we will aim to obtain a full numerical solution using the large scale computations.

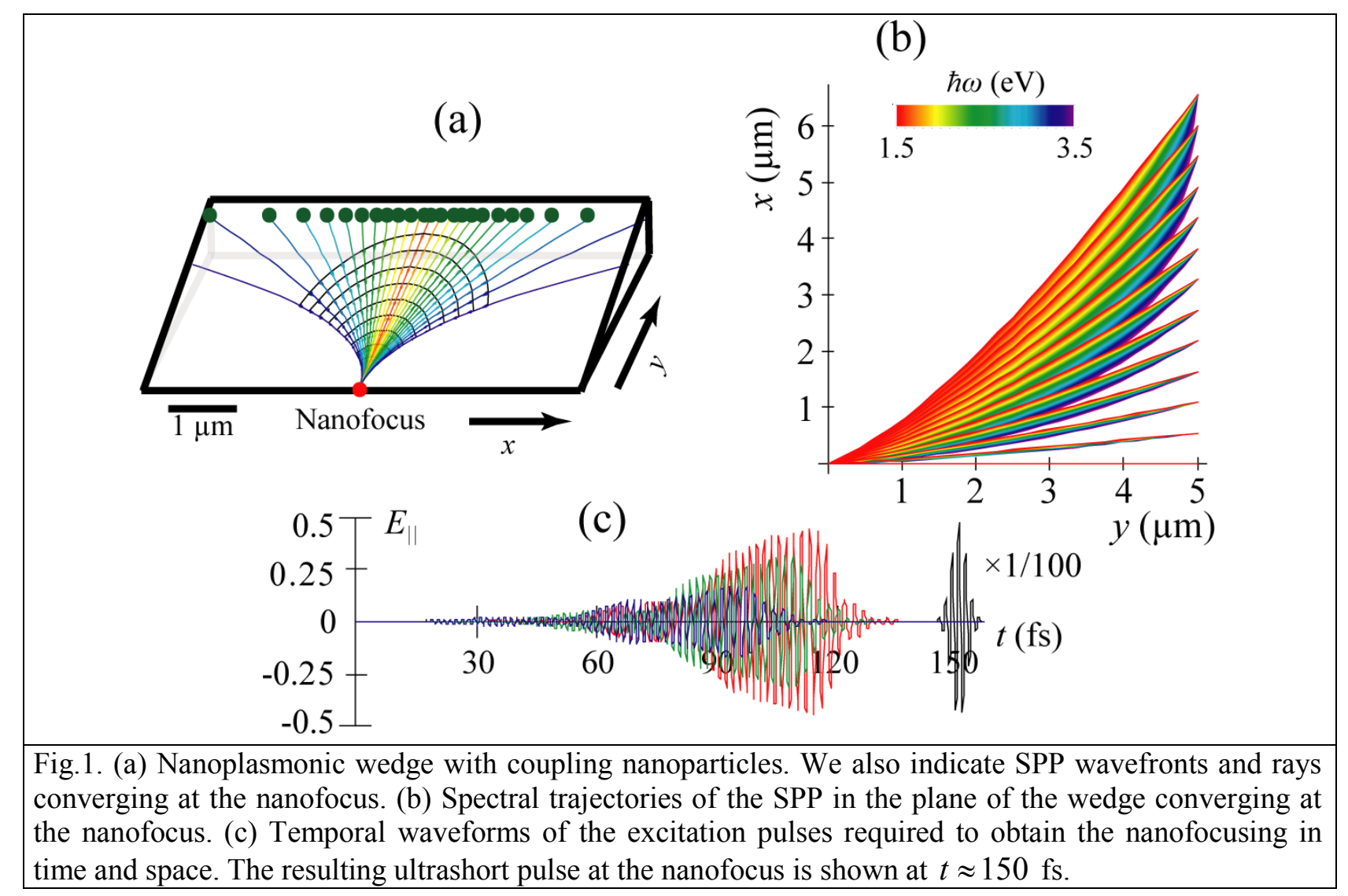

\subsection{SERS in Nanolenses [24]}

As an efficient nanolens, we have proposed a self-similar linear chain of several metal nanospheres with progressively decreasing sizes and separations [25]. The proposed system can be used for nanooptical detection, Raman characterization, nonlinear spectroscopy, nano-manipulation of single molecules or nanoparticles, and other applications. We have also computationally found the enhancement coefficient of the Surface Enhanced Raman Scattering (SERS) and have shown that it is considerably different from commonly used fourth power of the field enhancement [24].

\subsection{Computational Studies of SERS $[24,26]$}

We have revisited theory of one of the most important phenomena in nanoplasmonics, Surface Enhanced Raman Scattering [24, 26]. This theory shows that the predicted levels of enhancement in the red spectral region are still several orders of magnitude less than the enhancement factors $\sim 10^{13}-10^{14}$ observed experimentally. The difference may be due to the effects not taken into account by the theory: self-similar enhancement [25] or chemical enhancement [27]. We are planning further computational studies of SERS in 
systems with adiabatic compression that we have introduced earlier [20]. Note that recently the adiabatic compression effect that we predicted has been observed in two independent experiments [28, 29]. We trust that we can find a way to make SERS radiation much more intense by the better coupling between the far- and near-field zones that the adiabatic compression effect provides. This will provide unmatched sensitivity to SERS based methods of monitoring and detection of chemical and biological substances (threats).

\subsection{Excitation of Surface Plasmon Polaritons (SPPs) by Free Electron Impact [30]}

We have provided theoretical support and interpretation for the experimental investigation of the SPP generation by free-electron impact. This effect can be used as a basis of a novel method to visualize eigenmodes of plasmonic nanosystem by exciting them with an electron microscope beam.

\section{Publications Resulting from the Grant}

During the grant period of 2003-2007 we have published Refs. [1, 2, 19, 24, 30-37].

\section{Financials}

The allocated funds have been fully expended.

\section{References}

1. X. Li and M. I. Stockman, Time-Reversal Coherent Control in Nanoplasmonics, arXiv:0705.0553 (2007).

2. X. Li and M. I. Stockman, Coherent Control by Time-Reversal in Nanoplasmonics, Phys. Rev. Lett., (Submitted) (2007).

3. M. I. Stockman, S. V. Faleev, and D. J. Bergman, Coherent Control of Femtosecond Energy Localization in Nanosystems, Phys. Rev. Lett. 88, 67402-1-4 (2002).

4. M. I. Stockman, S. V. Faleev, and D. J. Bergman, Coherently Controlled Femtosecond Energy Localization on Nanoscale, Appl. Phys. B 74, S63-S67 (2002).

5. M. I. Stockman, S. V. Faleev, and D. J. Bergman, Coherently-Controlled Femtosecond Energy Localization on Nanoscale, Appl. Phys. B 74, 63-67 (2002).

6. M. I. Stockman, D. J. Bergman, and T. Kobayashi, in Proceedings of SPIE: Plasmonics: Metallic Nanostructures and Their Optical Properties, edited by N. J. Halas, Coherent Control of Ultrafast Nanoscale Localization of Optical Excitation Energy (SPIE, San Diego, California, 2003), Vol. 5221, p. 182-196.

7. M. I. Stockman, S. V. Faleev, and D. J. Bergman, in Ultrafast Phenomena XIII, CoherentlyControlled Femtosecond Energy Localization on Nanoscale (Springer, Berlin, Heidelberg, New York, 2003).

8. M. I. Stockman, D. J. Bergman, and T. Kobayashi, Coherent Control of Nanoscale Localization of Ultrafast Optical Excitation in Nanosystems, Phys. Rev. B 69, 054202-10 (2004).

9. M. I. Stockman and P. Hewageegana, Nanolocalized Nonlinear Electron Photoemission under Coherent Control, Nano Lett. 5, 2325-2329 (2005).

10. M. Sukharev and T. Seideman, Phase and Polarization Control as a Route to Plasmonic Nanodevices, Nano Lett. 6, 715-719 (2006).

11. M. Sukharev and T. Seideman, Coherent Control Approaches to Light Guidance in the Nanoscale, J. Chem. Phys. 124, 144707-1-8 (2006).

12. T. Brixner, F. J. G. d. Abajo, J. Schneider, C. Spindler, and W. Pfeiffer, Ultrafast Adaptive Optical near-Field Control, Physical Review B 73, 125437 (2006).

13. T. Brixner, F. J. G. d. Abajo, J. Schneider, and W. Pfeiffer, Nanoscopic Ultrafast Space-TimeResolved Spectroscopy, Phys. Rev. Lett. 95, 093901-1-4 (2005).

14. A. Kubo, K. Onda, H. Petek, Z. Sun, Y. S. Jung, and H. K. Kim, Femtosecond Imaging of Surface Plasmon Dynamics in a Nanostructured Silver Film, Nano Lett. 5, 1123-1127 (2005). 
15. A. Kubo, K. Onda, H. Petek, Z. Sun, Y. S. Jung, and H. K. Kim, in Ultrafast Phenomena XIV, edited by T. Kobayashi, T. Okada, T. Kobayashi, K. A. Nelson and S. D. Silvestri, Imaging of Localized Silver Plasmon Dynamics with Sub-Fs Time and Nano-Meter Spatial Resolution (Springer, Niigata, Japan, 2004), Vol. 79, p. 645-649.

16. P. v. d. Walle, L. Kuipers, and J. L. Herek, in Ultrafast Phenomena XV, Coherent Control of Light in Metal Nanostructures (Pacific Grove, California, 2006), p. Paper TuD3.

17. M. Bauer, D. Bayer, T. Brixner, F. J. G. d. Abajo, W. Pfeiffer, M. Rohmer, C. Spindler, and F. Steeb, in Ultrafast Phenomena XV, Adaptive Control of Nanoscopic Photoelectron Emission (Pacific Grove, California, 2006), p. Paper ThB3.

18. M. Aeschlimann, M. Bauer, D. Bayer, T. Brixner, F. J. G. d. Abajo, W. Pfeiffer, M. Rohmer, C. Spindler, and F. Steeb, Adaptive Subwavelength Control of Nano-Optical Fields, Nature 446, 301-304 (2007).

19. M. Durach, A. Rusina, K. Nelson, and M. I. Stockman, Toward Full Spatio-Temporal Control on the Nanoscale, Nano Lett. 7, (DOI:10.1021/n1071718g, 5 pages) (2007).

20. M. I. Stockman, Nanofocusing of Optical Energy in Tapered Plasmonic Waveguides, Phys. Rev. Lett. 93, 137404-1-4 (2004).

21. M. M. Wefers and K. A. Nelson, Ultrafast Optical Wave-Forms, Science 262, 1381-1382 (1993).

22. M. M. Wefers, K. A. Nelson, and A. M. Weiner, Multidimensional Shaping of Ultrafast Optical Waveforms, Opt. Lett. 21, 746-748 (1996).

23. T. Feurer, J. C. Vaughan, and K. A. Nelson, Spatiotemporal Coherent Control of Lattice Vibrational Waves, Science 299, 374-377 (2003).

24. K. Li, M. I. Stockman, and D. J. Bergman, Li, Stockman, and Bergman Reply to Comment on "Self-Similar Chain of Metal Nanospheres as an Efficient Nanolens", Phys. Rev. Lett. 97, 079702 (2006).

25. K. Li, M. I. Stockman, and D. J. Bergman, Self-Similar Chain of Metal Nanospheres as an Efficient Nanolens, Phys. Rev. Lett. 91, 227402-1-4 (2003).

26. M. I. Stockman (ed.) Surface Enhanced Raman Scattering - Physics and Applications (Springer-Verlag, Heidelberg New York Tokyo, 2006).

27. J. Jiang, K. Bosnick, M. Maillard, and L. Brus, Single Molecule Raman Spectroscopy at the Junctions of Large Ag Nanocrystals, J. Phys. Chem. B 107, 9964-9972 (2003).

28. E. Verhagen, L. Kuipers, and A. Polman, Enhanced Nonlinear Optical Effects with a Tapered Plasmonic Waveguide, Nano Lett. 7, 334-337 (2007).

29. C. Ropers, C. C. Neacsu, T. Elsaesser, M. Albrecht, M. B. Raschke, and C. Lienau, GratingCoupling of Surface Plasmons onto Metallic Tips: A Nano-Confined Light Source, Nano Lett. 7, 2784-2788 (2007).

30. M. V. Bashevoy, F. Jonsson, A. V. Krasavin, N. I. Zheludev, Y. Chen, and M. I. Stockman, Generation of Traveling Surface Plasmon Waves by Free-Electron Impact, Nano Lett. 6, 1113-1115 (2006).

31. M. I. Stockman, Slow Propagation, Anomalous Absorption, and Total External Reflection of Surface Plasmon Polaritons in Nanolayer Systems, Nano Lett. 6, 2604-2608 (2006).

32. M. Stockman (ed.) Electromagnetic Theory of SERS (Springer Verlag, 2006).

33. M. I. Stockman, K. Li, S. Brasselet, and J. Zyss, Octupolar Metal Nanoparticles as Optically Driven, Coherently Controlled Nanorotors, Chem. Phys. Lett. 433, 130-135 (2006).

34. M. I. Stockman, Criterion for Negative Refraction with Low Optical Losses from a Fundamental Principle of Causality, Phys. Rev. Lett. 98, 177404-1-4 (2007).

35. M. Durach, A. Rusina, K. Nelson, and M. I. Stockman, Toward Full Spatio-Temporal Control on the Nanoscale, arXiv:0705.0725 (2007).

36. M. I. Stockman and P. Hewageegana, Absolute Phase Effect in Ultrafast Optical Responses of Metal Nanostructures, Appl. Phys. A 89, 247-250 (2007). 
37. M. I. Stockman, M. F. Kling, U. Kleineberg, and F. Krausz, Attosecond Nanoplasmonic Field Microscope, Nat. Phot. 1, 539-544 (2007). 